\title{
Total glucosides of paeony (TGP) inhibits the production of inflammatory cytokines in oral lichen planus by suppressing the NF- $\mathrm{NB}$ signaling pathway
}

Yanni Wang ${ }^{1 *}$, Han Zhang ${ }^{2 *}$, Guanhuan $\mathrm{Du}^{1}$, Yufeng Wang ${ }^{1}$, Tianyi Cao ${ }^{1}$, Qingqiong Luo $^{2}$, Junjun Chen ${ }^{1}$, Fuxiang Chen ${ }^{2 * *}$, Guoyao Tang ${ }^{1 * *}$.

${ }^{1}$ Department of Oral Medicine, Shanghai Ninth People's Hospital, Shanghai Jiao Tong University School of Medicine, 639 Zhi Zao Ju Road, Shanghai 200011, China.

${ }^{2}$ Department of Clinical Immunology, Shanghai Ninth People's Hospital, Shanghai Jiao Tong University School of Medicine, 639 Zhi Zao Ju Road, Shanghai 200011, China.

${ }^{* *}$ Corresponding authors: Guoyao Tang, tel +86 21 23271699-5696, e-mail address: tanggy@shsmu.edu.cn and Fuxiang Chen, tel +86 21 63080932, e-mail address: chenfx@ sjtu.edu.cn

*Both authors contributed equally to this work. 


\section{Abstract}

Total glucosides of paeony (TGP) is a bioactive compound extracted from paeony roots and has been widely used to ameliorate inflammation in several autoimmune and inflammatory diseases. However, the anti-inflammatory effect of TGP on oral lichen planus (OLP), a chronic inflammatory oral condition characterized by T-cell infiltration and abnormal epithelial keratinization cycle remains unclear. In this study, we found that TLR4 was highly expressed and activation of the NF- $\mathrm{BB}$ signaling pathway was obviously observed in the OLP tissues. Moreover, there was significant higher mRNA expression of inflammatory cytokines interleukin-6 (IL-6) and tumor necrosis factor- $\alpha(\mathrm{TNF}-\alpha)$ in OLP keratinocytes than normal oral epithelial keratinocytes. With the help of the cell culture model by stimulating the keratinocyte HaCaT cells with lipopolysaccharides (LPS), we mimicked the local inflammatory environment of OLP. And we further confirmed that TGP could inhibit LPS-induced production of IL-6 and TNF- $\alpha$ in HaCaT cells via a dose-dependent manner. TGP treatment decreased the phosphorylation of $\mathrm{I} \kappa \mathrm{B} \alpha$ and $\mathrm{NF}-\kappa \mathrm{B}$ p65 proteins, thus leading to less nuclear translocation of NF- $\mathrm{kB}$ p65 in HaCaT cells. Therefore, our data suggested that TGP may be a new potential candidate for the therapy of OLP.

Keywords: total glucosides of paeony; oral lichen planus; TLR4; NF-кB.

\section{Introduction}

As a chronic autoimmune, mucocutaneous disease, lichen planus (LP) usually affects the oral and genital mucosa, skin, scalp and nails[1]. It occurs more commonly 
1 in women aged over 30 years; however, incidence in children has also been

2 reported[2, 3]. Oral mucosal lesions appear in 50 to $70 \%$ of LP patients and may be

3 exclusive in 20 to $30 \%$ of them[4]. The occurrence of oral lichen planus (OLP) ranges

4 from 0.5 to $4 \%$, and the prevalence of malignant transformation from 0 to $5.3 \%[4,5]$.

5 The etiology of OLP is still unclear, but immunological processes are believed to play

6 critical roles[6]. Cytotoxic $\mathrm{CD}^{+} \mathrm{T}$ cells are directed against basilar keratinocytes,

7 which leads to the degeneration and lyses of basal cells[7]. Cytokines such as IFN- $\gamma$,

8 TNF- $\alpha$, GM-SF and IL-6 are released, provoking a local inflammatory response thus

9 further exacerbating the tissue damage[8].

OLP may present in two main types: a reticular type which requires no treatment

11 or an atrophic/erosive type which does need therapy because of its burning sensation, severe pain and malignant transformation tendency[9]. So far, treatments of OLP include corticosteroids drugs, immunosuppressive agents, photodynamic therapy and cryosurgery etc[4]. However, as inflammatory condition in OLP often recurs, surgery is not first recommended. And the associated side effects and complications like secondary candida infection of oral mucosa and suppression of adrenal function with corticosteroids drugs; local irritation, mucosal pigmentation and the risk of promoting the occurrence of squamous-cell carcinoma by affecting the MAPK and p53 signaling with immunosuppressive agents; nausea, dizziness, paresthesia and headache with photodynamic therapy, are disadvantageous $[4,5]$. Therefore, the identification of novel and effective therapeutic agents for OLP is essential. 
1 have attracted extensive attention on their treatment for numerous diseases. One of

2 them is total glucosides of paeony (TGP), the bioactive compound extracted from

3 paenoy roots. Now, TGP has been proved to be protective in several animal disease

4 models. Wu et al. reported that TGP relieved early renal injury through inhibiting the

5 secretion of inflammatory factors like intracellular adhesion molecule-1 (ICAM-1),

6 IL-1 and TNF- $\alpha$ in the kidneys of diabetic rats[10]. TGP could also modulate serum

7 cholesterol and triglyceride levels and suppress TNF- $\alpha$, IL-6 expression thus

8 attenuated the development of atherosclerotic disease[11]. Moreover, TGP minimized

9 damages in rheumatoid arthritis by inhibiting Th1/Th17 cells differentiation and

10 fibroblast-like synoviocytes proliferation [12-14]. However, the effect of TGP in OLP

11 has not yet been elucidated.

In the present study, we investigated the effect of TGP on OLP keratinocytes.

\section{Materials and methods}

\subsection{Immunohistochemistry assay}

Human OLP specimens were obtained from patients diagnosed with OLP at the Department of Oral Medicine, Shanghai Ninth People's Hospital. Normal oral mucosal tissues were collected from the Department of Oral and Maxillofacial Surgery from 2014 to 2015. Signed informed consent forms were acquired before biopsy and pathological examination. All experimental procedures were ethically 
1 approved by the Independent Ethics Committee of Shanghai Ninth People's Hospital

2 (No. 2014069). Specimens were fixed and embedded with paraffin. Sections of the

3 specimens were blocked with 5\% BSA and incubated with anti-TLR4 (1:100, Abcam,

4 MA, USA) and anti-NF- $\kappa$ B p65 (1:100, Cell Signaling, MA, USA) antibodies

5 overnight at $4^{\circ} \mathrm{C}$. Next, slides were washed and incubated with second antibodies

6 conjugated with peroxidase (Dako, CA, USA) for $1 \mathrm{~h}$. Then, slides were developed

7 with 3, 3'-Diaminobenzidine (Dako, CA, USA) substrate and counter-stained with

8 hematoxylin. After dehydration, slides were visualized under Axio Imager (Zeiss,

9 Oberkonchen, Germany).

\subsection{Quantitative real-time PCR (qRT-PCR)}

Human OLP specimens $(n=27)$ and normal oral mucosal tissues $(n=14)$ were

digested using $0.25 \%$ dispase II (Stemcell, CAN) at $4^{\circ} \mathrm{C}$ for $12 \mathrm{~h}$. Characteristics of the patients with OLP were shown in Table 1.The epithelium and subepithelial layers were separated by sterile muscle forceps. The isolated epithelium was digested in $0.25 \%$ trypsin for 8 min to obtain a single-cell suspension of keratinocytes. TRIzol reagent (Invitrogen, CA, USA) was used to extract total RNA from keratinocytes. cDNA was reverse transcribed from up to $1 \mu \mathrm{g}$ total RNA using a PrimeScript ${ }^{\mathrm{TM}} \mathrm{RT}$ reagent kit (Takara, Kyoto, Japan). mRNA expression was determined by qRT-PCR assay using the Applied Biosystems 7500 Real-Time PCR System (Life techologies, CA, USA). The following PCR conditions were used: $95^{\circ} \mathrm{C}$ for $5 \mathrm{~min}$, followed by 40 cycles of $95^{\circ} \mathrm{C}$ for $15 \mathrm{~s}$ and $60^{\circ} \mathrm{C}$ for $1 \mathrm{~min}$, completed with a melt cure analysis. 
1 Relative gene expression was determined by the $2^{-\Delta \Delta C T}$ method and was calculated

2 using $\beta$-actin as the reference gene. Sequences of PCR primer pairs were given in 3 Table 2.

4

\subsection{Cell line and culture}

The HaCaT cell line was provided by China Infrastructure of Cell Line Resources (Beijing, China). HaCaT cells were maintained in Dulbecco's modified Eagle's medium (DMEM) (Gibco, CA, USA) supplemented with 10\% FBS (Gibco, $\mathrm{CA}, \mathrm{USA}$ ) and penicillin-streptomycin (Gibco, CA, USA) in a humidified $5 \% \mathrm{CO}_{2}$ incubator at $37^{\circ} \mathrm{C}$. To simulate a local immune microenvironment of OLP, the culture medium was replaced with keratinocyte serum free medium (KSFM) (Gibco, CA, USA) in which HaCaT cells were cultured overnight and then stimulated with LPS (Sigma Aldrich, MO, USA) at a concentration of $10 \mu \mathrm{g} / \mathrm{ml}$.

\subsection{Enzyme-linked immunosorbent assay (ELISA)}

HaCaT cells were seeded in a 96-well plate $\left(5 \times 10^{4}\right.$ cells per well $)$ and cultured in KSFM for $24 \mathrm{~h}$. Then cells were stimulated by TGP (Liwah Plant Extract Technology, Ningbo, China) at serial concentrations $(1,2.5,5$, and $10 \mu \mathrm{g} / \mathrm{mL})$ in KSFM for $2 \mathrm{~h}$ and then treated by LPS $(10 \mu \mathrm{g} / \mathrm{mL})$ for $48 \mathrm{~h}$. Supernatants were collected to measure the concentration of IL- 6 and TNF- $\alpha$ by ELISA (eBioscience, CA, USA) assays according to the manufacturer's instructions. 


\subsection{Cell viability assay}

To investigate the toxicity of TGP, CCK-8 (Dojindo, Kumamoto, Japan) assay was applied to assess cell viability. HaCaT cells were seeded in a 96-well plate (5000 cells per well) and treated with increasing concentrations of TGP in DMEM for $24 \mathrm{~h}$ or $48 \mathrm{~h}$. Then, $10 \mu \mathrm{l}$ of CCK-8 reagent were added to each well. Wells containing incubation media without cells were incubated in parallel with test samples to measure the absorbance background. After incubation for $1.5 \mathrm{~h}$, absorbance values were measured at $450 \mathrm{~nm}$.

\subsection{Western blot}

HaCaT cells were treated with increasing concentrations of TGP $(0,1,2.5,5$, and $10 \mu \mathrm{g} / \mathrm{ml})$ for $2 \mathrm{~h}$, followed by LPS incubation $(10 \mu \mathrm{g} / \mathrm{mL})$ in the continued presence of TGP for another $2 \mathrm{~h}$. The cells were lysed with RIPA buffer (Beyotime, Shanghai, China) containing phosphatase inhibitor cocktail (Roche, Germany) and phenylmethanesulfonylfluoride (PMSF). The total cell lysates were centrifuged and the supernatants were used for western blotting. Equal quantities of protein samples were separated by $10 \%$ sodium dodecyl sulfate-polyacrylamide gel electrophoresis. and transferred to polyvinylidene difluoride (PVDF) membranes. The PVDF membranes were blocked with $5 \%$ nonfat milk for $1 \mathrm{~h}$ at room temperature and incubated overnight at $4^{\circ} \mathrm{C}$ with appropriate primary antibodies (1:1000):

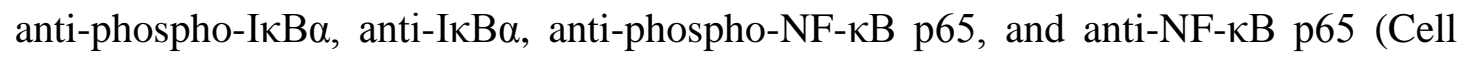
Signaling, MA, USA); anti- $\beta$-actin (Sigma-Aldrich, Mo, USA). After washed by 
1 TBST, the membranes were incubated with HRP-conjugated secondary antibodies

2 (1:5000) (Cell Signaling, MA, USA). Labeled proteins were visualized using ECL

3 reagent (Merck Millipore, USA). The protein levels were quantified by Image-Pro

4 Plus 6.0 (Media Cybernetics, CA, USA).

\subsection{Immunofluorescence assay}

HaCaT cells deposited on glass slides were fixed with $4 \%$ paraformaldehyde for 20 min. After permeabilized with $0.2 \%$ Triton X-100 for 6 min and blocked with $1 \%$ bovine serum albumin for $40 \mathrm{~min}$, the slides were treated with anti-NF- $\mathrm{BB}$ p65 (1:400) antibody at $4^{\circ} \mathrm{C}$ overnight. Anti-rabbit IgG labeled with Alexa Fluor 488 $(1: 1000)$ served as the secondary antibody. After washing three times, sections were stained with DAPI for 3 min to visualize the nuclei. Slides were examined using an inverted microscope equipped with fluorescence optics (Olympus, Osaka, Japan).

\subsection{Statistical analysis}

Each experiment was repeated at least three times. The Student's $t$ test was used to analyze the statistical significance between two groups. Statistical differences were considered significant when value of $\mathrm{p}<0.05$.

\section{Results}

\subsection{Expression of TLR4 and NF-אB p65 in OLP keratinocytes}

Since OLP is a long-lasting inflammatory process, some studies recently reported 
that keratinocytes play a critical role in maintaining the inflammatory environment of OLP through TLR4/NF-kB activation [15-17]. To verify the local inflammatory environment of OLP, immunohistochemical staining was performed to analyze the expression of TLR4 and NF- $\mathrm{BB}$ p65 in OLP specimens. As shown in Fig. 1, both TLR4 and NF- $\kappa$ B p65 were highly expressed in keratinocytes of OLP lesions compared with normal oral mucosal tissues, which confirmed the significant role of TLR4/NF- $\mathrm{B}$ signaling pathway in pathogenesis of OLP.

\subsection{Expression of IL-6 and TNF- $\alpha$ in OLP keratinocytes}

The production of inflammatory cytokines should be increased when the NF- $\kappa \mathrm{B}$ inflammation signaling pathway is activated. To observe the levels of inflammatory cytokines in OLP, 27 OLP specimens and 14 normal control mucosal tissues were digested with dispase II and trypsin to release keratinocytes. The mRNA expression of IL-6 and TNF- $\alpha$ in keratinocytes was measured by qRT-PCR assay. As shown in Fig. 2, expression of IL-6 and TNF- $\alpha$ mRNA was increased in OLP keratinocytes compared to keratinocytes obtained from normal mucosal tissues. These results, together with the data shown in Fig.1, suggested that TLR4 was highly expressed and NF- $\kappa \mathrm{B}$ inflammatory signaling pathway was activated in OLP keratinocytes.

\subsection{TGP inhibition of IL-6 and TNF- $\alpha$ production in LPS-treated HaCaT cells}

Following stimulation by exogenous factors such as TLR4 ligand LPS, keratinocytes actively secrete a large amount of cytokines. HaCaT cells, the 
1 immortalized human skin keratinocytes, are useful substitutes for oral keratinocytes

2 [15]. Therefore experiments were performed on HaCaT cells with LPS stimulation to

3 mimic the immune microenvironment of OLP lesions [15]. To determine if LPS

4 stimulation of $\mathrm{HaCaT}$ cells simulates the inflammatory environment in OLP, the

5 levels of NF-kB-dependent inflammatory cytokines were measured by ELISA. After

6 stimulating $\mathrm{HaCaT}$ cells with LPS $(10 \mu \mathrm{g} / \mathrm{ml})$ in $\mathrm{KSFM}$ for $48 \mathrm{~h}$, we found that

7 production of IL-6 and TNF- $\alpha$ was markedly increased (Fig. 3). The data suggested

8 that the mimic inflammatory responses could to some extent reflect the immune

9 environment in OLP.

To investigate the anti-inflammatory effect of TGP, HaCaT cells were

11 pre-incubated with different concentration of TGP for $2 \mathrm{~h}$, followed by stimulating

12 with LPS for an additional $48 \mathrm{~h}$. The levels of IL-6 and TNF- $\alpha$ in cell culture 13 supernatants were measured by ELISA assay. As shown in Fig. 3, secretion of IL-6 14 and TNF- $\alpha$ was significantly decreased in a dose-related fashion with the increase in 15 TGP concentrations. This result suggested that TGP could reduce the LPS-induced cytokines.

\subsection{TGP did not affect the proliferation of HaCaT cells}

To further study the effect of TGP on keratinocytes, we first investigated if TGP affected HaCaT cell proliferation using the CCK-8 assay. HaCaT cells were cultured with increasing concentrations of TGP for 24 or $48 \mathrm{~h}$. CCK-8 was then added to the 
1 culture medium for $1.5 \mathrm{~h}$, followed by measurement of absorbance values at $450 \mathrm{~nm}$

2 as an indicator of $\mathrm{HaCaT}$ cell viability. We found no obvious change in the

3 proliferation of HaCaT cells, which suggested that TGP was non-toxic at doses of

$4 \quad 1-10 \mu \mathrm{g} / \mathrm{ml}$ under culture conditions (Fig. 4).

\subsection{TGP suppression of LPS-induced activation of the NF- $\kappa B$ inflammatory}

\section{signaling pathway in $\mathrm{HaCaT}$ cells}

$\mathrm{NF}-\mathrm{\kappa B}$ activation of keratinocytes is critically involved in the progression of OLP and involves the production of inflammatory cytokines. To study the anti-inflammatory mechanism of TGP, we investigated its effect on LPS-induced NF- $\kappa \mathrm{B}$ activation in $\mathrm{HaCaT}$ cells. After treating $\mathrm{HaCaT}$ cells with increasing amounts of TGP, LPS $(10 \mu \mathrm{g} / \mathrm{ml})$ was added to the incubation medium and cellular protein was extracted for western blot assay. As shown in Figs. 5A and 5B, expression of

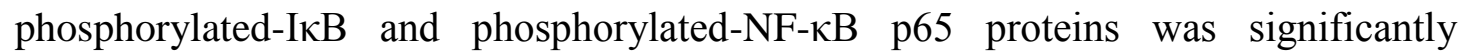
decreased in a dose-related fashion with elevating concentrations of TGP, with no change in total $\mathrm{I} \kappa \mathrm{B} \alpha$, total $\mathrm{NF}-\kappa \mathrm{B}$ p65 and $\beta$-actin. These results indicated that the

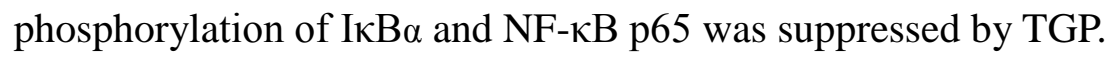

Immunofluorescence assays were then conducted to examine the effect of TGP on nuclear translocation of NF- $\kappa \mathrm{B}$ p65. As shown in Fig. 5C, LPS-stimulated HaCaT

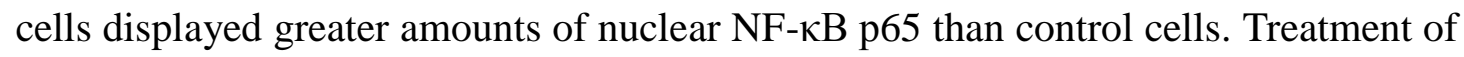
HaCaT cells with $10 \mu \mathrm{g} / \mathrm{ml}$ TGP reduced the intensity of NF- $\kappa \mathrm{B}$ p65 nuclear staining, indicating that TGP had suppressed nuclear translocation of NF- $\mathrm{B}$ p65. The above 
results shown also suggested that TGP could inhibit the activation of NF- $\mathrm{BB}$ inflammatory signaling pathway in a mimic OLP local immune environment in vitro.

\section{Discussion}

Our findings demonstrated that activation of TLR4/NF- $\kappa B$ pathway was detected in OLP tissue. Moreover, the expression of inflammatory cytokines IL- 6 and TNF- $\alpha$ was significantly up-regulated in OLP keratinocytes. In this study, we found that TGP markedly inhibited the inflammatory response of the mimic OLP by suppressing the secretion of inflammatory cytokine IL- 6 and TNF- $\alpha$. The inhibiting effect of TGP may be attributed to its suppression of NF- $\kappa \mathrm{B}$ signaling pathway.

OLP is a common autoimmune mucocutaneous disease of unknown etiology, characterized by a T-cell-mediated immune process against keratinocytes leading to keratinocytes damage and subepithelial band-like infiltration of T lymphocytes[18-20]. In the early stages of disease development, the keratinocytes are target cells to suffer apoptosis. Once active, however, those keratinocytes have the ability to amplify an inflammatory signal by producing inflammatory mediators and recruiting inflammatory cells that can mediate the development of OLP, as well as favor the disease chronicity[21, 22]. These findings confirmed the critical role of keratinocytes in the pathological process in OLP lesions.

Recently, the role of keratinocytes in sustaining the inflammatory environment of OLP has attracted extensive attention, one critical possible pathogenesis of which is NF- $\kappa \mathrm{B}$ activation. Previous studies demonstrated that NF- $\kappa \mathrm{B}$ was highly expressed on 
1 basal and suprabasal keratinocytes of the OLP lesions [17, 23], which was in positive

2 correlation with the degree of cytotoxic T cells infiltration [17]. As an inflammatory

3 regulator, $\mathrm{NF}-\mathrm{\kappa B}$ could be activated by multiple inflammatory signals, one crucial

4 pathway was triggering of toll-like receptors (TLRs). Recently, Janardhanam SB et

5 al.[24] and Siponen M et al.[16] demonstrated the high expression of TLR4 in OLP

6 epithelium. In accordance with previous studies, our data indicated that both TLR4

7 and NF-kB p65 were highly expressed in keratinocytes of OLP lesions, verifying the

8 critical role of TLR4/NF-kB activation in OLP pathogenesis. Moreover, some articles

9 reported that $\mathrm{NF}-\kappa \mathrm{B}$ associated cytokines played important roles in the exacerbation

10 and perpetuation of OLP. Recently, the elevated levels of NF- $\kappa \mathrm{B}$-dependent

11 inflammatory cytokines such as IL-1 $\beta$, IL-6, IL-8, and TNF- $\alpha$ were detected in both

12 salivary and serum of OLP patients $[25,26]$. In our study, we found that mRNA levels

13 of IL-6 and TNF- $\alpha$ in keratinocytes of OLP lesions were significantly increased,

14 suggesting that keratinocytes might maintain the inflammatory response of OLP

15 through activating TLR4/NF- $\kappa B$ signaling pathway and secreting IL- 6 and TNF- $\alpha$.

However, due to the pathological condition of OLP keratinocytes and severe oral

17 fungal infection of OLP patients, the primary culture and its passage always turn out

failed. So far, there is no appropriate model to study the pathogenesis of OLP. HaCaT

19 cells are immortalized human skin keratinocytes and frequently substituted for oral

20 keratinocytes in many studies due to their easy growth and infinite proliferation

21 [27-29]. LPS, the ligand of TLR4, has long been used as a mediator to induce

22 inflammatory response. Therefore, we used LPS-induced HaCaT cell inflammatory 
responses to simulate the local immune environment of OLP in the current study. After stimulating with LPS, the production of inflammatory cytokines IL-6 and TNF- $\alpha$ was markedly increased, this demonstrated that our cell model was proved to well reflect the inflammatory environment of OLP.

TGP is extracted from the roots of Paeonia lactiflora and has been widely used in the therapy for autoimmune disease in Chinese medicine. It is reported that TGP has anti-inflammatory, hepatoprotective $[30,31]$ and immuno-regulatory activities. Recently, the anti-inflammatory effect of TGP has been attracting increasing attention. In our study, we demonstrated that TGP has marked therapeutic effect in the mimic OLP local immune environment by reducing the production of IL- 6 and TNF- $\alpha$ from LPS-treated HaCaT cells. Based on our results and others, IL-6 and TNF- $\alpha$ released by keratinocyte from OLP lesions are likely contribute to the pathogenesis of OLP. As primary cytokines, IL- 6 and TNF- $\alpha$ can induce proliferation and differentiation of T cells, meanwhile, they also upregulate adhesion molecules expression and increase $\mathrm{T}$ cell adhesion which may contribute to T cell infiltration in OLP[32]. Furthermore, previous studies reported the high increase of inflammatory cytokines in salivary from OLP patients, and the change of which could be used for monitoring the therapeutic response[33]. Therefore, the anti-inflammatory effect of TGP in mimic OLP offered the possibilities of a therapeutic drug for OLP through suppressing secretion of IL-6 and TNF- $\alpha$ from keratinocyte.

Subsequently, we further investigated the mechanisms by which TGP suppress inflammatory responses in the mimic OLP. NF- $\mathrm{B}$ is an important inflammatory 
1 mediators which plays critical roles in regulating inflammatory signaling pathway

2 [34]. It has been confirmed that in the NF- $\mathrm{BB}$ inflammatory signal pathway, NF- $\mathrm{B}$

$3 \mathrm{p} 50 / \mathrm{p} 65$ and $\mathrm{I} \kappa \mathrm{B}$ tripolymer is inactive in the cytoplasm. Upon activation, I $\mathrm{KB}$ is

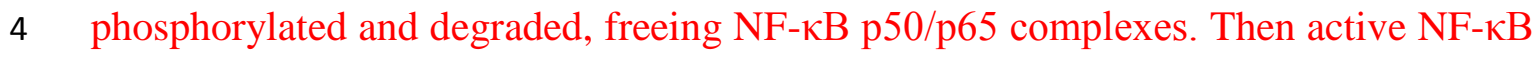

5 p65 translocate to the nucleus, either alone or in combination with other transcription

6 factors, where it induces target gene expression including genes encoding

7 proinflammatory cytokines[35]. In our experiment, we demonstrated that NF- $\kappa \mathrm{B}$

8 signaling pathway was activated in mimic OLP by using the model of LPS-induced

9 HaCaT cell inflammatory responses. Therefore, we speculated whether anti-inflammatory effect of TGP was related to the inhibition of NF- $\kappa \mathrm{B}$ signaling

11 pathway. As a result, TGP could obviously decrease the phosphorylation of $\mathrm{I} \kappa \mathrm{B} \alpha$ and

$12 \mathrm{NF}-\kappa \mathrm{B}$ p65, thus leading to less nuclear translocation of NF- $\kappa \mathrm{B}$ p65. Our results 13 suggested that the anti-inflammatory mechanism of TGP in a mimic OLP local 14 immune environment is mediated by suppressing the NF- $\kappa \mathrm{B}$ signaling pathway. simulate the local OLP immune environment and found TGP has a potentially 17 therapeutic effect on OLP. Furthermore, the anti-inflammatory mechanism of TGP is associated with inhibition of NF- $\mathrm{BB}$ signaling pathway leading to reduced secretion of 19 inflammatory cytokines. The results of our study suggest new opportunities to use TGP in treatment of OLP. 
2 Clinical Key Specialized Department (No. [2013]544) and National Science

3 Foundation of China (Grant No. 81170967). The authors thank Department of Oral and Maxillofacial Surgery, Shanghai Ninth People's Hospital for their help of normal oral mucosal tissues collection.

\section{Conflict of interest}

The authors declare no financial or commercial conflicts of interest.

\section{References}

[1] R.J. Krupaa, S.L. Sankari, K.M. Masthan, E. Rajesh, Oral lichen planus: An overview, Journal of pharmacy \& bioallied sciences, 7 (2015) S158-161.

[2] S. Gupta, M.K. Jawanda, Oral Lichen Planus: An Update on Etiology, Pathogenesis, Clinical Presentation, Diagnosis and Management, Indian journal of dermatology, 60 (2015) 222-229.

[3] R. Laeijendecker, T. Van Joost, B. Tank, A.P. Oranje, H.A. Neumann, Oral lichen planus in childhood, Pediatric dermatology, 22 (2005) 299-304.

[4] M.M. Nico, J.D. Fernandes, S.V. Lourenco, Oral lichen planus, Anais brasileiros de dermatologia, 86 (2011) 633-641; quiz 642-633.

[5] S.B. Ismail, S.K. Kumar, R.B. Zain, Oral lichen planus and lichenoid reactions: etiopathogenesis, diagnosis, management and malignant transformation, Journal of oral science, 49 (2007) 89-106.

[6] H. Malekzadeh, M. Robati, H. Yousefimanesh, M. Ghafourian Boroujerdnia, R. Nadripour, Salivary Interferon Gamma and Interleukin-4 Levels in Patients Suffering from Oral Lichen Planus, Cell journal, 17 (2015) 554-558.

[7] S. Jimson, N. Balachader, N. Anita, R. Babu, Immunologically mediated oral diseases, Journal of pharmacy \& bioallied sciences, 7 (2015) S209-212.

[8] R. Lu, J. Zhang, W. Sun, G. Du, G. Zhou, Inflammation-related cytokines in oral lichen planus: an overview, Journal of oral pathology \& medicine : official publication of the International Association of Oral Pathologists and the American Academy of Oral Pathology, 44 (2015) 1-14.

[9] G. Shailaja, J.V. Kumar, P.V. Baghirath, U. Kumar, G. Ashalata, A.B. Krishna, Estimation of malignant transformation rate in cases of oral epithelial dysplasia and lichen planus using immunohistochemical expression of Ki-67, p53, BCL-2, and BAX markers, Dental research journal, 12 (2015) 235-242.

[10] Y. Wu, K. Ren, C. Liang, L. Yuan, X. Qi, J. Dong, J. Shen, S. Lin, Renoprotective effect of total glucosides of paeony (TGP) and its mechanism in experimental diabetes, Journal of pharmacological sciences, 109 (2009) 78-87. 
[11] J. Li, C.X. Chen, Y.H. Shen, Effects of total glucosides from paeony (Paeonia lactiflora Pall) roots on experimental atherosclerosis in rats, Journal of ethnopharmacology, 135 (2011) 469-475.

[12] W. Zhang, S.M. Dai, Mechanisms involved in the therapeutic effects of Paeonia lactiflora Pallas in rheumatoid arthritis, International immunopharmacology, 14 (2012) 27-31.

[13] J. Lin, L. Xiao, G. Ouyang, Y. Shen, R. Huo, Z. Zhou, Y. Sun, X. Zhu, J. Zhang, B. Shen, N. Li, Total glucosides of paeony inhibits Th1/Th17 cells via decreasing dendritic cells activation in rheumatoid arthritis, Cellular immunology, 280 (2012) 156-163.

[14] X.Y. Jia, Y. Chang, X.J. Sun, H.X. Wu, C. Wang, H.M. Xu, L. Zhang, L.L. Zhang, Y.Q. Zheng, L.H. Song, $W$. Wei, Total glucosides of paeony inhibit the proliferation of fibroblast-like synoviocytes through the regulation of $\mathrm{G}$ proteins in rats with collagen-induced arthritis, International immunopharmacology, 18 (2014) 1-6.

[15] Y. Ge, Y. Xu, W. Sun, Z. Man, L. Zhu, X. Xia, L. Zhao, Y. Zhao, X. Wang, The molecular mechanisms of the effect of Dexamethasone and Cyclosporin A on TLR4 /NF-kappaB signaling pathway activation in oral lichen planus, Gene, 508 (2012) 157-164.

[16] M. Siponen, J.H. Kauppila, Y. Soini, T. Salo, TLR4 and TLR9 are induced in oral lichen planus, Journal of oral pathology \& medicine : official publication of the International Association of Oral Pathologists and the American Academy of Oral Pathology, 41 (2012) 741-747.

[17] A. Santoro, A. Majorana, E. Bardellini, S. Festa, P. Sapelli, F. Facchetti, NF-kappaB expression in oral and cutaneous lichen planus, The Journal of pathology, 201 (2003) 466-472.

[18] L. Baccaglini, K. Thongprasom, M. Carrozzo, M. Bigby, Urban legends series: lichen planus, Oral diseases, 19 (2013) 128-143.

[19] D. Farhi, N. Dupin, Pathophysiology, etiologic factors, and clinical management of oral lichen planus, part I: facts and controversies, Clinics in dermatology, 28 (2010) 100-108.

[20] M.R. Roopashree, R.V. Gondhalekar, M.C. Shashikanth, J. George, S.H. Thippeswamy, A. Shukla, Pathogenesis of oral lichen planus--a review, Journal of oral pathology \& medicine : official publication of the International Association of Oral Pathologists and the American Academy of Oral Pathology, 39 (2010) 729-734.

[21] M.R. Payeras, K. Cherubini, M.A. Figueiredo, F.G. Salum, Oral lichen planus: focus on etiopathogenesis, Archives of oral biology, 58 (2013) 1057-1069.

[22] P.B. Sugerman, N.W. Savage, L.J. Walsh, Z.Z. Zhao, X.J. Zhou, A. Khan, G.J. Seymour, M. Bigby, The pathogenesis of oral lichen planus, Critical reviews in oral biology and medicine : an official publication of the American Association of Oral Biologists, 13 (2002) 350-365.

[23] G. Zhou, K. Xia, G.F. Du, X.M. Chen, X.Y. Xu, R. Lu, H.M. Zhou, Activation of nuclear factor-kappa B correlates with tumor necrosis factor-alpha in oral lichen planus: a clinicopathologic study in atrophic-erosive and reticular form, Journal of oral pathology \& medicine : official publication of the International Association of Oral Pathologists and the American Academy of Oral Pathology, 38 (2009) 559-564.

[24] S.B. Janardhanam, S. Prakasam, V.T. Swaminathan, K.N. Kodumudi, S.L. Zunt, M. Srinivasan, Differential expression of TLR-2 and TLR-4 in the epithelial cells in oral lichen planus, Archives of oral biology, 57 (2012) 495-502.

[25] Y. Zhang, M. Lin, S. Zhang, Z. Wang, L. Jiang, J. Shen, J. Bai, F. Gao, M. Zhou, Q. Chen, NF-kappaB-dependent cytokines in saliva and serum from patients with oral lichen planus: a study in an ethnic Chinese population, Cytokine, 41 (2008) 144-149.

[26] N.L. Rhodus, B. Cheng, S. Myers, W. Bowles, V. Ho, F. Ondrey, A comparison of the 
pro-inflammatory, NF-kappaB-dependent cytokines: TNF-alpha, IL-1-alpha, IL-6, and IL-8 in different oral fluids from oral lichen planus patients, Clinical immunology (Orlando, Fla.), 114 (2005) 278-283. [27] K. Avezov, A.Z. Reznick, D. Aizenbud, Oxidative damage in keratinocytes exposed to cigarette smoke and aldehydes, Toxicology in vitro : an international journal published in association with BIBRA, 28 (2014) 485-491.

[28] K. Moharamzadeh, R. Van Noort, I.M. Brook, A.M. Scutt, Cytotoxicity of resin monomers on human gingival fibroblasts and HaCaT keratinocytes, Dental materials : official publication of the Academy of Dental Materials, 23 (2007) 40-44.

[29] M. Li, F. Gao, Z.S. Zhou, H.M. Zhang, R. Zhang, Y.F. Wu, M.H. Bai, J.J. Li, S.R. Lin, J.Y. Peng, Arecoline inhibits epithelial cell viability by upregulating the apoptosis pathway: implication for oral submucous fibrosis, Oncology reports, 31 (2014) 2422-2428.

[30] N. Xiang, X.M. Li, M.J. Zhang, D.B. Zhao, P. Zhu, X.X. Zuo, M. Yang, Y. Su, Z.G. Li, Z. Chen, X.P. Li, Total glucosides of paeony can reduce the hepatotoxicity caused by Methotrexate and Leflunomide combination treatment of active rheumatoid arthritis, International immunopharmacology, 28 (2015) 802-807.

[31] Z. Chen, X.P. Li, Z.J. Li, L. Xu, X.M. Li, Reduced hepatotoxicity by total glucosides of paeony in combination treatment with leflunomide and methotrexate for patients with active rheumatoid arthritis, International immunopharmacology, 15 (2013) 474-477.

[32] T. Yamamoto, T. Osaki, Characteristic cytokines generated by keratinocytes and mononuclear infiltrates in oral lichen planus, The Journal of investigative dermatology, 104 (1995) 784-788.

[33] N.L. Rhodus, B. Cheng, S. Myers, L. Miller, V. Ho, F. Ondrey, The feasibility of monitoring NF-kappaB associated cytokines: TNF-alpha, IL-1alpha, IL-6, and IL-8 in whole saliva for the malignant transformation of oral lichen planus, Molecular carcinogenesis, 44 (2005) 77-82.

[34] P.J. Barnes, M. Karin, Nuclear factor-kappaB: a pivotal transcription factor in chronic inflammatory diseases, The New England journal of medicine, 336 (1997) 1066-1071.

[35] A.S. Baldwin, Jr., The NF-kappa B and I kappa B proteins: new discoveries and insights, Annual review of immunology, 14 (1996) 649-683.

\section{Figure Legends}

Fig. 1. Expression of TLR4 and NF- $\mathrm{BB}$ p65 analyzed by immunohistochemical staining in keratinocytes of OLP and healthy control tissue. TLR4 were stained brown and were detected in the membrane and cytoplasm of $\mathrm{HC}$ (A) and OLP (C) keratinocytes; NF-кB p65 were stained dark brown and were detected in cytoplasm of HC (B) and OLP (D) keratinocytes. Ten sections were observed and pictures were representative of enlarged images (200x). 
1 Fig. 2. Levels of IL-6 and TNF- $\alpha$ evaluated by qRT-PCR in keratinocytes from 27

2 OLP patients and 14 healthy control subjects. (A) mRNA expression levels of IL-6. (B)

3 mRNA expression levels of TNF- $\alpha$. Data are presented as mean \pm SD. $* p<0.05$.

Fig. 3. TGP inhibition of LPS-induced IL-6 and TNF- $\alpha$ secretion in HaCaT cells. HaCaT cells were treated with TGP $(0,1,2.5,5$, and $10 \mu \mathrm{g} / \mathrm{ml})$ for $2 \mathrm{~h}$, followed by addition of LPS $(10 \mu \mathrm{g} / \mathrm{ml})$ for $48 \mathrm{~h}$. Culture medium was analyzed for cytokine levels by ELISA. (A) level of IL-6 in cell culture supernatant. (B) level of TNF- $\alpha$ in cell culture supernatant. Results are representative of three independent experiments and are presented as mean \pm SD. \#\# $\mathrm{p}<0.01$ (\# VS cells without LPS treatment); * $\mathrm{p}$ $<0.05, * * \mathrm{p}<0.01$ (*VS LPS-stimulated cells without TGP treatment).

Fig. 4. CCK-8 assay of potential cytotoxic effects of TGP $(1-10 \mu \mathrm{g} / \mathrm{ml})$ at $24 \mathrm{~h}$ or $48 \mathrm{~h}$ in $\mathrm{HaCaT}$ cells. Data are presented as mean $\pm \mathrm{SD}$.

Fig. 5. TGP suppression of the NF- $\kappa B$ inflammatory signaling pathway in LPS-treated $\mathrm{HaCaT}$ cells. (A) western blot assay was performed to investigate expression of $\mathrm{NF}-\kappa \mathrm{B}$ inflammatory signaling proteins. $\beta$-actin was monitored as a control for protein loading. (B) quantitative analysis of phospho-IкB $\alpha$ (left panel) and phospho-NF-кB p65 (right panel) protein expression. Data are presented as mean \pm SD. \# $\mathrm{p}<0.05$, \#\# $\mathrm{p}<0.01$ (\# VS cells without LPS treatment); $* \mathrm{p}<0.05$, ** $\mathrm{p}<0.01$, $* * * \mathrm{p}<0.001$ (* VS LPS-stimulated cells without TGP treatment). (C) nuclear 
1 translocation of NF-кB p65 was visualized under fluorescence microscopy. (a), (d),

2 and (g), staining of nuclei (blue); (b), (e), and (h), detection of NF-kB p65 (green); (c),

3 a merge of (a) and (b); (f), a merge of (d) and (e); (i), a merge of (g) and (h). 
Table 1

Characteristics of the patients with OLP and HC

\begin{tabular}{cccccc}
\hline & $\begin{array}{c}\text { Number of } \\
\text { cases }\end{array}$ & $\begin{array}{c}\text { Age range } \\
\text { (years) }\end{array}$ & $\begin{array}{c}\text { Averge age } \\
\text { (years) }\end{array}$ & $\begin{array}{c}\text { Number of } \\
\text { females }\end{array}$ & $\begin{array}{c}\text { Number of } \\
\text { males }\end{array}$ \\
\hline HC & 14 & $19-49$ & $38.2 \pm 7.62$ & 9 & 5 \\
OLP & 27 & $21-61$ & $40.3 \pm 10.57$ & 18 & 9 \\
\hline
\end{tabular}


Table 2

The primers for qRT-PCR analysis.

\begin{tabular}{ll}
\hline Gene & Primer sequence 5'-3' \\
\hline IL-6 & F-CCTGAACCTTCCAAAGATGGC \\
& R-TTCACCAGGC AAGTCTCCTCA \\
TNF- $\alpha$ & F-CCTCTCTCTAATCAGCCCT CTG \\
& R-GAGGACCTGGGAGTAGAT GAG \\
\multirow{2}{*}{-actin } & F-CCTGGCACCCAGCACAAT \\
& R-GGGCCGGACTCGTCATACT \\
\hline
\end{tabular}


Figure. 1

$\mathrm{HC}$
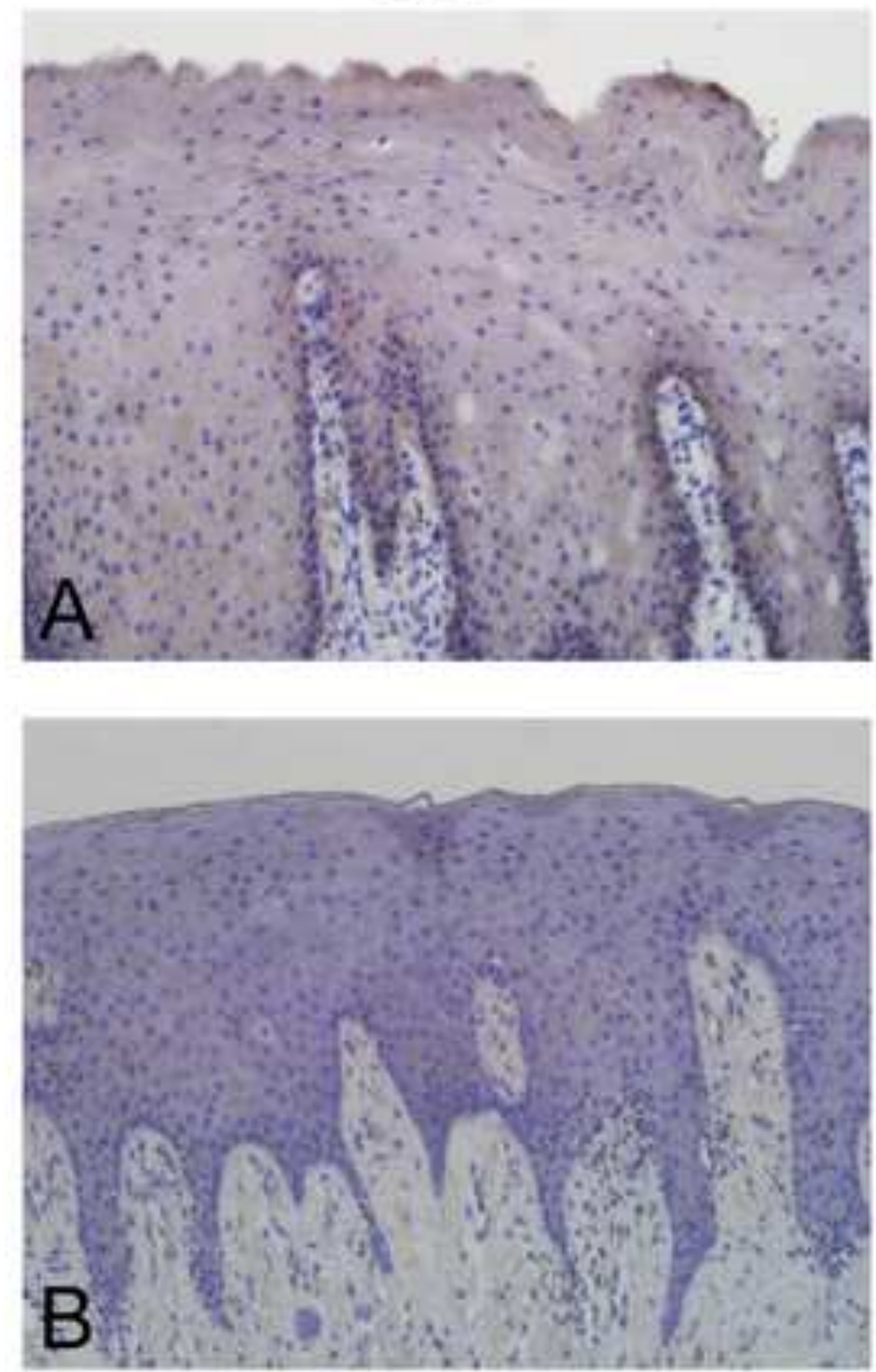

OLP

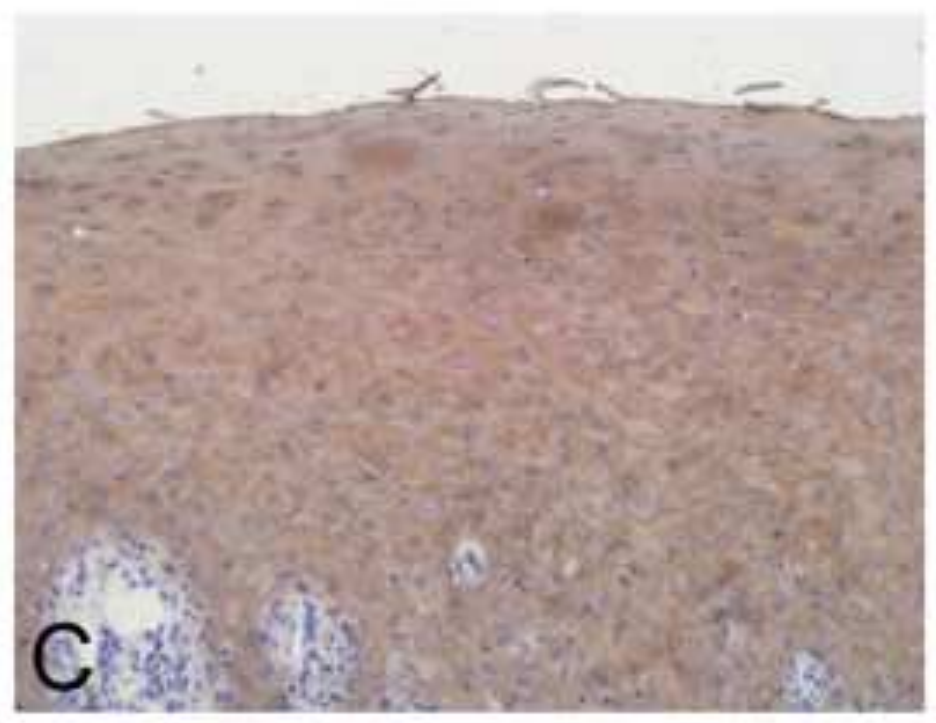

TLR4

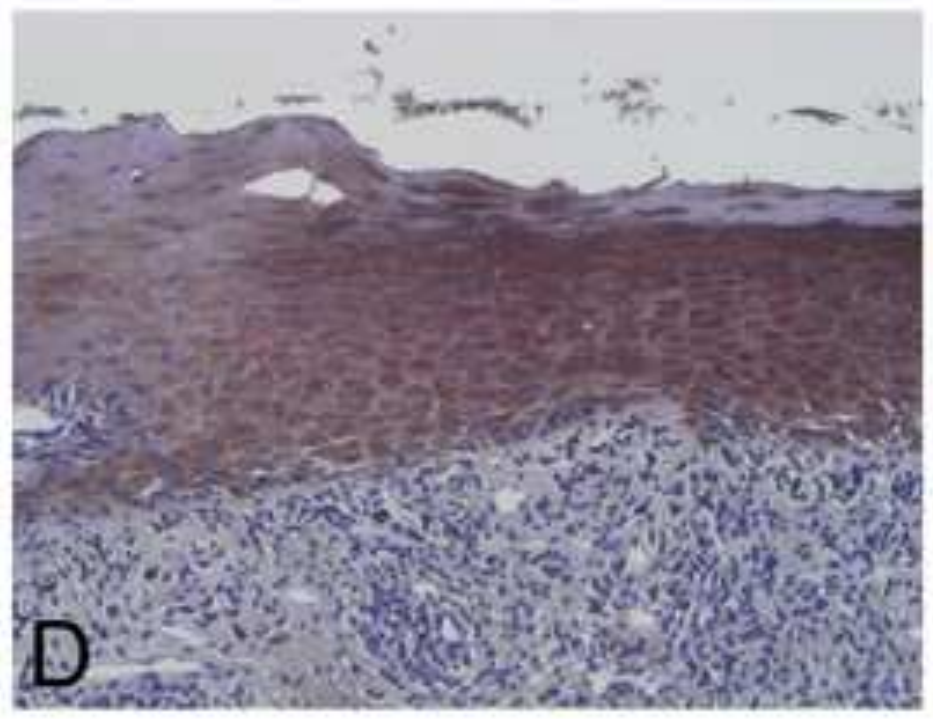

NF-kB p65 
Figure. 2

A
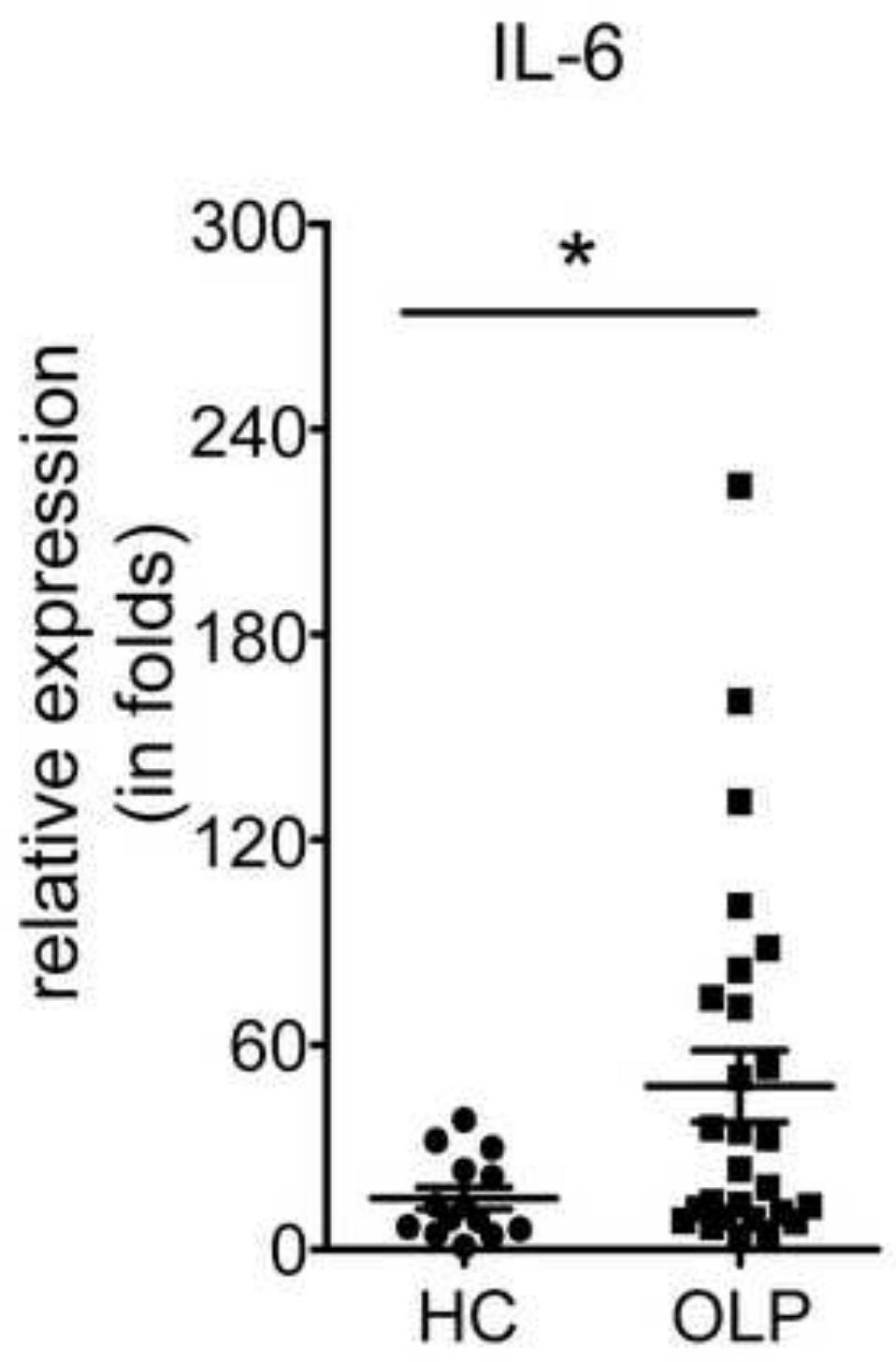

B

TNF- $\alpha$

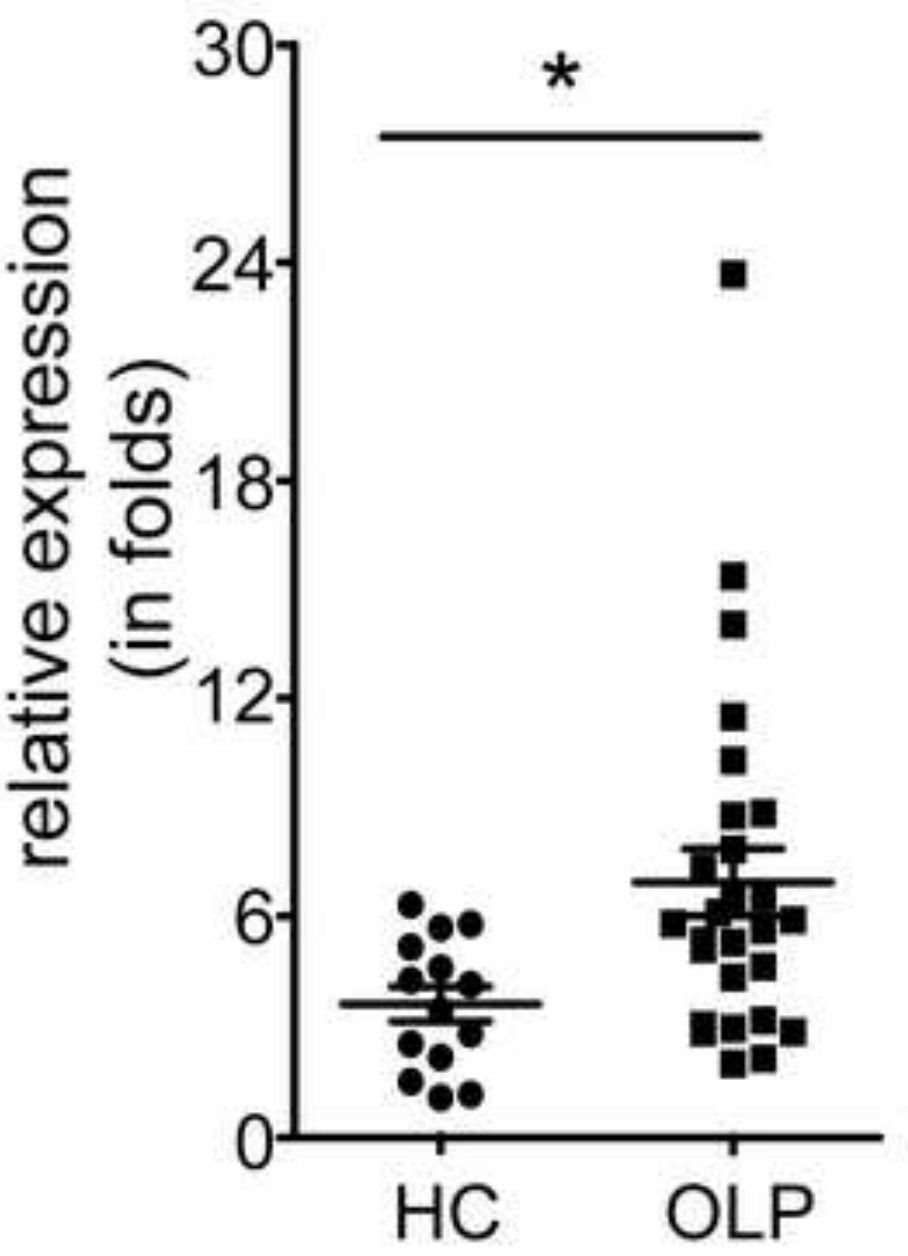


Figure. 3
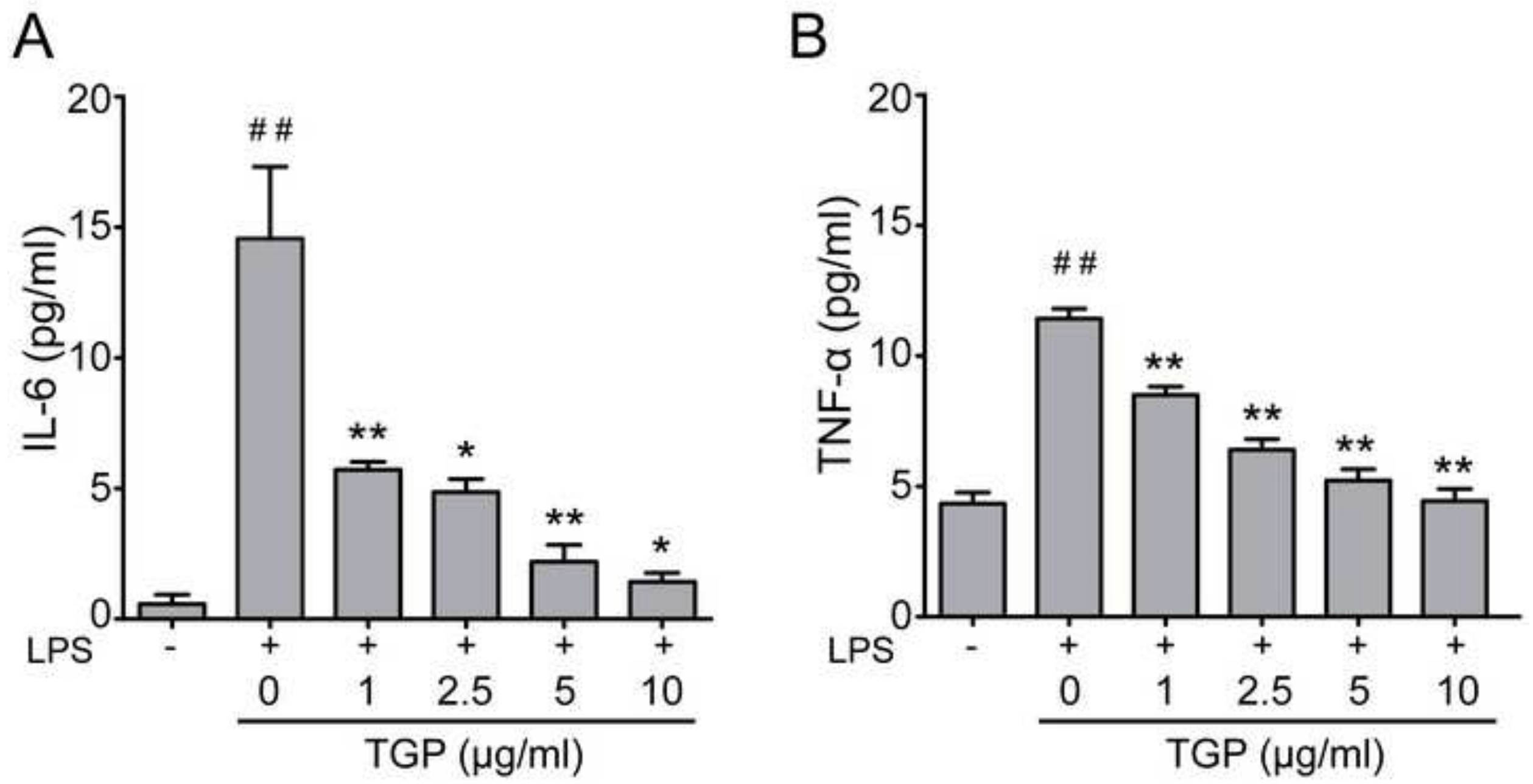
Figure. 4

A

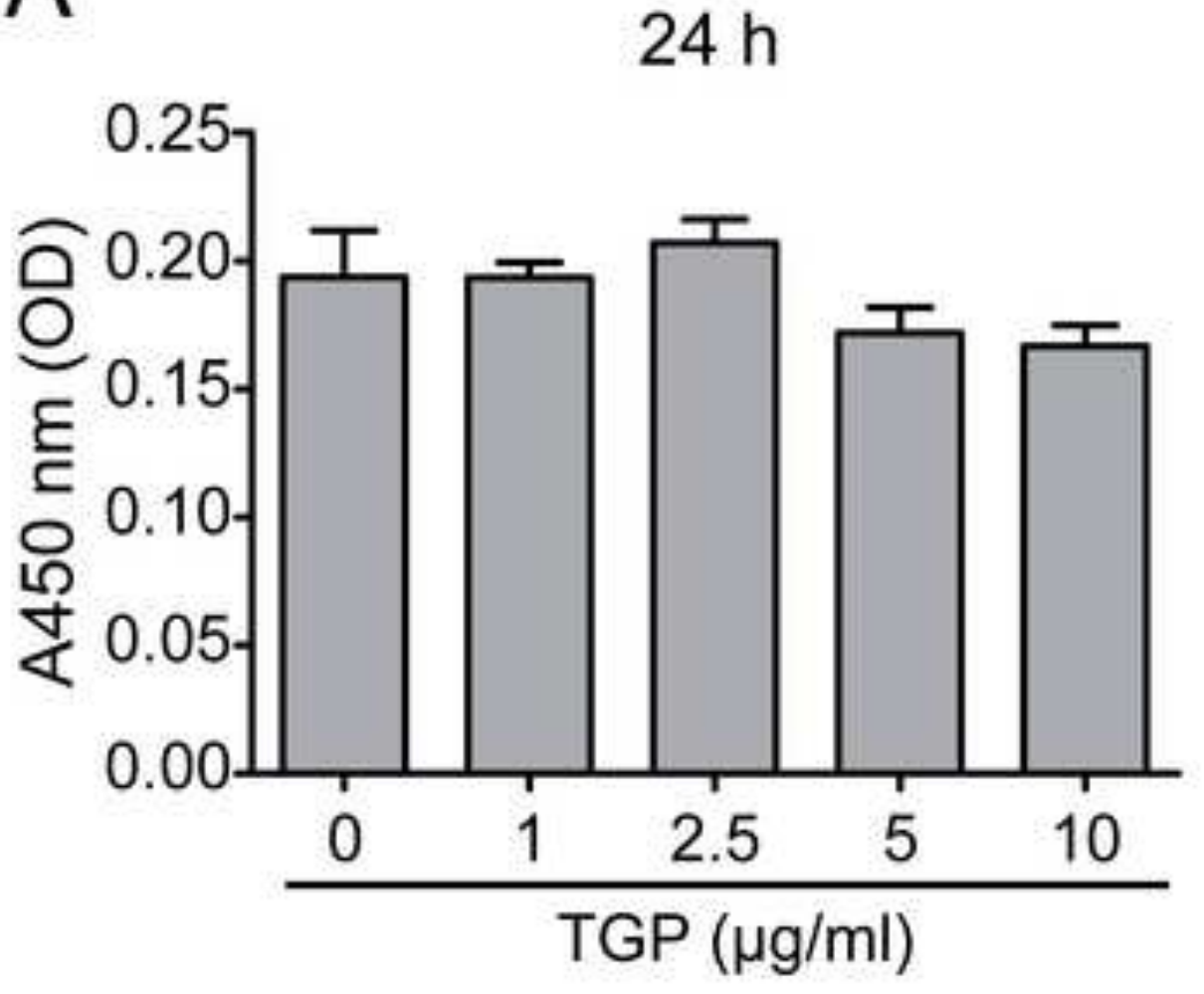

B

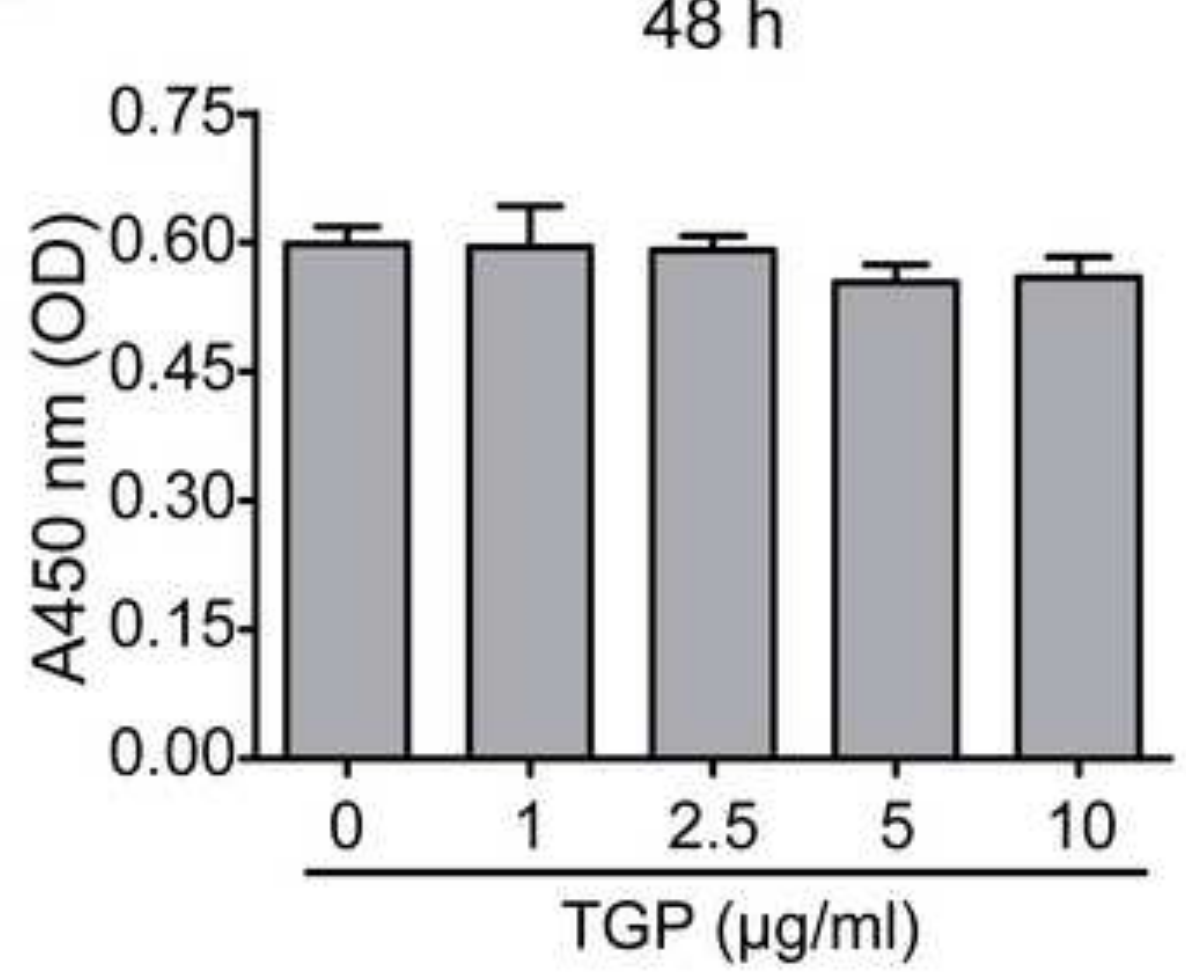


Figure. 5

A

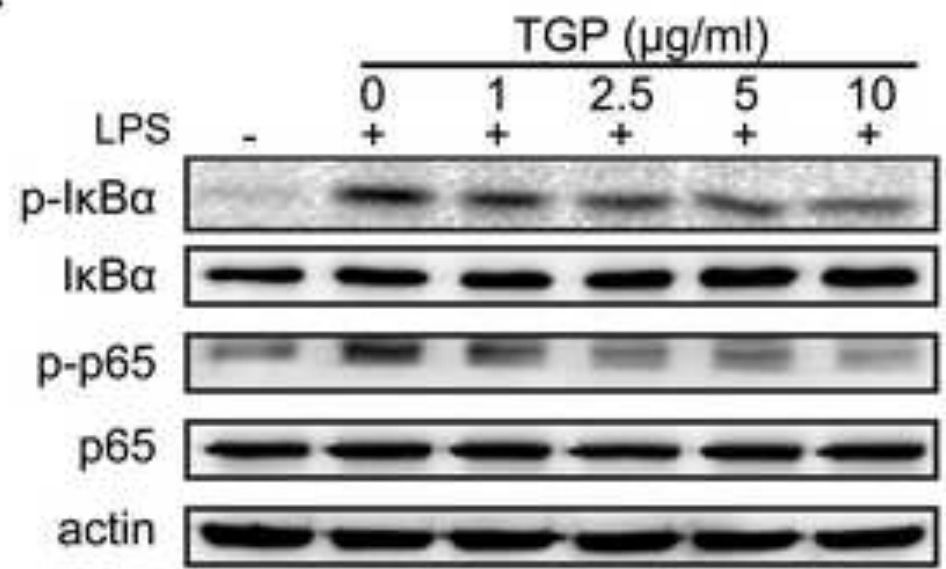

B
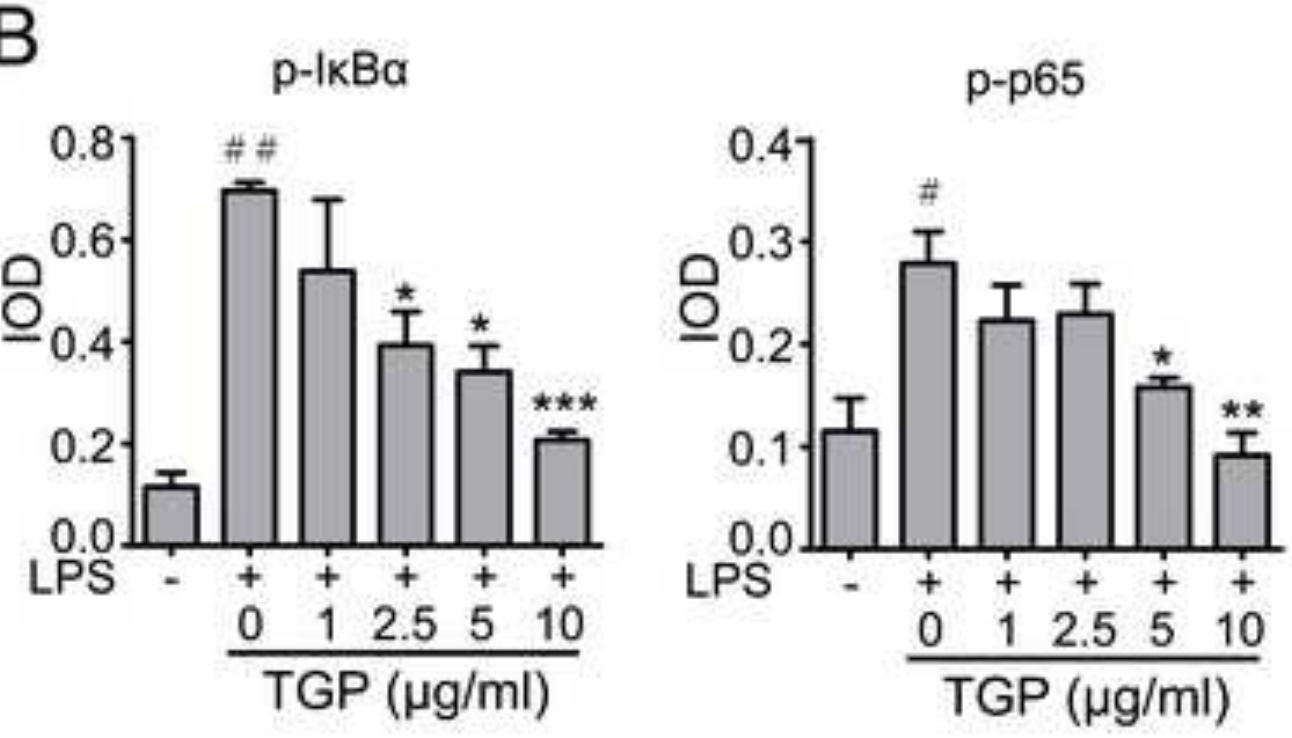

C

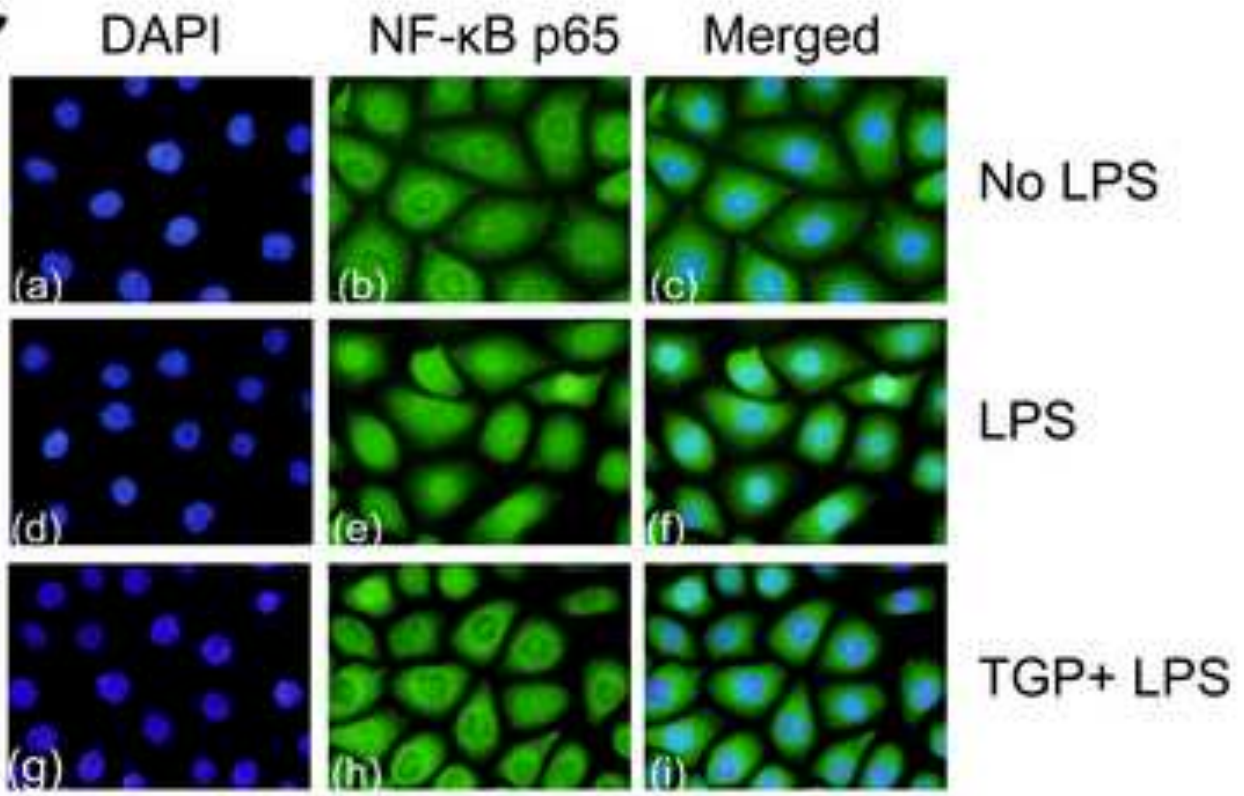

